\title{
a Companion?
}

\section{Anna Cadoret}

The terminology "companion" (the English translation of the French "camarade") refers to a celebrated conjecture formulated by Deligne in his foundational paper "Weil II" [Del80, Conj. (1.2.10)]. This conjecture lies at the intersection of Grothendieck's theory of motives and the Langlands program; it reflects the expectation that every finitedimensional continuous $\overline{\mathbb{Q}}_{\ell}$-representation of the absolute Galois group of a finitely generated field $K$ of characteristic $p \neq \ell$ satisfying (very!) mild assumptions arises from geometry in the sense that it is cut out by an algebraic correspondence on the $\overline{\mathbb{Q}}_{\ell}$-cohomology group of a variety $X$ over $K$. In particular, such a representation should admit, for every prime $\ell^{\prime} \neq p$, a $\overline{\mathbb{Q}}_{\ell^{\prime}}$-companion, namely the representation cut out by the same algebraic correspondence on the $\overline{\mathbb{Q}}_{\ell^{\prime}}$-cohomology group of $X$. For function fields of smooth varieties over a finite field, it is now a theorem. In contrast, its number field analogue, the Fontaine-Mazur conjecture [FM95], is still widely open.

Companions and motives. The story begins with the strategy elaborated by Grothendieck to prove the Weil conjectures [Wei49]. Fix a finite field $k$ of characteristic $p$ and for every integer $n$, let $k_{n}$ denote its degree- $n$ extension. A $k$-Weil number of weight $w \in \mathbb{Z}$ is an algebraic number whose complex conjugates all have complex absolute value $|k|^{\frac{w}{2}}$. The Weil conjectures give precise estimates for the number $N_{n}(X)$ of $k_{n}$-points on a smooth projective variety $X$ of dimension $d$ over $k$. This is expressed in terms of the zêta function $Z_{X}(T)=\exp \left(\sum_{n \geq 1} N_{n} \frac{T^{n}}{n}\right)$ as follows:

Anna Cadoret is a professor of mathematics at IMJ-PRG, Sorbonne Université, and a junior member of the Institut Universitaire de France. Her email address is anna.cadoret@imj-prg.fr.

Communicated by Notices Associate Editor William McCallum.

For permission to reprint this article, please contact:

reprint-permission@ams.org.

DOI: https://doi.org/10.1090/noti2308 there exists $P_{1}(T), \ldots, P_{2 d}(T) \in \mathbb{Z}[T]$ such that $P_{0}(T)=1-T$, $P_{2 d}(T)=1-|k|^{d} T$, and

[Rationality] $Z_{X}(T)=\prod_{1 \leq w \leq 2 d} P_{w}(T)^{(-1)^{w+1}} ;$

[Riemann hypothesis] the roots of $P_{w}(T)$ are $k$-Weil numbers of weight $w$;

[Funct. equation] $Z_{X}\left(\frac{1}{|k|^{d} T}\right)=\epsilon|k|^{\frac{n \chi(X)}{2}} Z_{X}(T)$, with $\chi(X)=\sum_{1 \leq w \leq 2 d}(-1)^{w} \operatorname{deg}\left(P_{w}(T)\right), \epsilon= \pm 1$.

What is hidden behind the formulation of the Weil conjectures is the Lefschetz formula in algebraic topology, which counts the number of fixed points of a continuous endomorphism $F: X \rightarrow X$ on a compact topological space $X$ as the alternating sum of its traces $\sum_{w \geq 0} \operatorname{Tr}\left(F^{*} \mid H_{\text {sing, } w}(X, \mathbb{Q})\right)$ on the singular homology groups of $X$. Observing that $N_{n}(X)$ is the number of fixed points of the $n$th power Frobenius endomorphism $F: X \rightarrow X$ of $X$ on $X(\bar{k})$, Weil had the bright intuition that there should exist a good cohomology theory for smooth projective varieties over $k$ that could play the part of singular cohomology. In particular, the $P_{w}(T)$ appearing in the expression of $Z_{X}(T)$ should be the inverse characteristic polynomials of $F$ acting on these prospective cohomology groups.

The first part of the strategy was thus to construct a cohomology theory for varieties over fields of characteristic $p>0$ satisfying the usual axioms of singular cohomology (Kunneth formula, Poincaré duality, existence of cycle class maps, etc.); such a good cohomology theory is now called a Weil cohomology theory. This part of the problem was solved by Grothendieck and his school during the 60s with the formidable construction of étale cohomology and its by-product $\ell$-adic cohomology. The existence of $\ell$-adic cohomology was enough to solve the Weil conjectures except for the Riemann hypothesis, which can now be reformulated by saying that the roots of $\operatorname{det}\left(1-T F^{*} \mid H^{w}\left(X_{\bar{k}}, \overline{\mathbb{Q}}_{\ell}\right)\right)$ are $k$-Weil numbers of weight $w$. 
To prove the Riemann hypothesis, it would be enough to show that the normalized Frobenius $|k|^{-\frac{w}{2}} F^{*}$ arises from an anti-autoadjoint operator on a $\mathbb{Q}$-vector space $H$ equipped with a positive definite bilinear form and such that $H \otimes \mathbb{Q}_{\ell}=H^{w}\left(X_{\bar{k}}, \mathbb{Q}_{\ell}\right)$. This observation led Grothendieck to formulate a coherent net of conjecturesthe standard conjectures-on algebraic cycles, from which the above property of the normalized Frobenius should follow straightforwardly [Kle68].

While the Riemann hypothesis was eventually proved by Deligne using a wonderful combination of geometric and analytic methods (relying deeply on the properties of $\ell$-adic cohomology!) [Del74], the standard conjectures remain widely open. But this does not alter their significance, which goes far beyond providing a conceptual proof of the Riemann hypothesis. In the first place they reposition the original problem in a broader setting-the one of pure motives, which can be roughly regarded as an attempt to linearize the category $\operatorname{SmP}(K)$ of smooth projective varieties over a field $K$. More precisely, Grothendieck constructed a natural pseudo-abelian $\mathbb{Q}$-linear $\otimes$-category $\mathrm{Mot}_{K}$ - the category of pure motives over $K$-which comes with a "linearization" $\otimes$-functor $\mathfrak{h}: \operatorname{SmP}(K) \rightarrow \operatorname{Mot}_{K}$. Assuming the standard conjectures, the category $\operatorname{Mot}_{K}$ is polarizable (hence semisimple), Tannakian, and, for every Weil cohomology theory $H: \operatorname{SmP}(K) \rightarrow \operatorname{Vect}_{Q}$ with coefficients in a characteristic 0 field $Q$, endowed with a $Q$ linear "realization" fiber functor $\bar{H}: \operatorname{Mot}_{K} \otimes_{\mathbb{Q}} Q \rightarrow$ Vect $_{Q}$ such that $\bar{H} \circ \mathfrak{h}=H: \operatorname{SmP}(K) \rightarrow \operatorname{Vect}_{Q}$. In particular, the "linearization" $\otimes$-functor $\mathfrak{h}: \operatorname{SmP}(K) \rightarrow \operatorname{Mot}_{K}$ can also be regarded as a "universal Weil cohomology" functor.

An important feature of Weil cohomologies is that they usually factor through a natural "enriched" Tannakian subcategory $\mathcal{T}_{H} \hookrightarrow$ Vect $_{Q}$. E.g.,

(1) for $K=\mathbb{C}$ and singular cohomology $H=H_{\text {sing }}$ : $\operatorname{SmP}(K) \rightarrow \operatorname{Vect}_{\mathbb{Q}}, \mathcal{T}_{H_{\text {sing }}}$ is the category $\mathbb{Q}$-PHS of $\mathbb{Q}$ rational polarizable Hodge structures;

(2) for $\ell$-adic cohomology $H=H_{\ell}: \operatorname{SmP}(K) \rightarrow$ Vect $_{\mathbb{Q}_{\ell}}$, $\mathcal{T}_{H_{\ell}}$ is the category $\operatorname{Rep}_{\mathbb{Q}_{\ell}}\left(\pi_{1}(K)\right)$ of finite-dimensional $\mathbb{Q}_{\ell^{-}}$ vector spaces equipped with a continuous action of the absolute Galois group $\pi_{1}(K)$ of $K$.

As $\bar{H}: \operatorname{Mot}_{K} \bigotimes_{\mathbb{Q}} Q \rightarrow \operatorname{Vect}_{Q}$ induces an equivalence onto its essential image $\mathcal{T}_{H}^{\text {ess }}$, understanding $\operatorname{Mot}_{K}$ essentially amounts to understanding $\mathcal{T}_{H}^{\text {ess }}$. This may sound tautological since Grothendieck's construction of $\mathrm{Mot}_{K}$ tells us exactly what $\mathcal{T}_{H}^{e s s}$ should be. Namely, the morphisms should be those induced by algebraic correspondences and the objects should be those cut out by algebraic correspondences on the $Q$-vector spaces $H(X)$ for $X \in$ $\operatorname{SmP}(K)$. However, this description is not really useful because we know almost nothing about algebraic correspondences. The expected miracle is that, however, $\mathcal{T}_{H}^{\text {ess }}$ can be easily described as a subcategory of $\mathcal{T}_{H}$, at least under suitable assumptions on $K$. For morphisms, one expects $\bar{H}: \operatorname{Mot}_{K} \otimes_{\mathbb{Q}} Q \rightarrow \mathcal{T}_{H}$ to be fully faithful. This is a fancy way to restate what is called, in (1) and when $K=\mathbb{C}$, the Hodge conjecture, and in (2) and when $K$ is finitely generated, the Tate conjecture. In (2), there is an additional issue due to the fact that $\mathrm{Mot}_{K}$ is conjecturally semisimple while $\operatorname{Rep}_{\mathbb{Q}_{\ell}}\left(\pi_{1}(K)\right)$ is not. So the latter should be replaced by the full subcategory $\operatorname{Rep}_{\mathbb{Q}_{\ell}}\left(\pi_{1}(K)\right)^{s s} \hookrightarrow \operatorname{Rep}_{\mathbb{Q}_{\ell}}\left(\pi_{1}(K)\right)$ of semisimple representations of $\pi_{1}(K)$. On the other hand, $\mathcal{T}_{H}^{\text {ess }}$ should contain the $\pi_{1}(K)$-representations $H^{w}\left(X_{\bar{K}}, \mathbb{Q}_{\ell}\right)$ for $X \in \operatorname{SmP}(K)$ but the semisimplicity of these is still a widely open conjecture.

Anyway, assuming it, what remains to describe are the simple objects in $\mathcal{T}_{H}^{\text {ess }}$ or, as it is more convenient to work with coefficients in an algebraically closed field, in $\mathcal{T}_{H}^{\text {ess }} \otimes \bar{Q}$. This is the problem the companion conjecture answers (implicitly) in the situation of (2) when $K$ has characteristic $p>0$, i.e., is the function field $K=\kappa(\eta)$ of a smooth, geometrically irreducible variety $S$ over a finite field $k$, with generic point $\eta$.

Fix a prime $\ell \neq p$. From the construction of $\operatorname{Mot}_{K}$, the simple objects in $\mathcal{T}_{H_{\ell}}^{\text {ess }} \otimes \overline{\mathbb{Q}}_{\ell}$ are $\pi_{1}(K)$-subquotients $V_{\ell}$ of $H^{i}\left(X_{\bar{K}}, \overline{\mathbb{Q}}_{\ell}(j)\right)$ for $X \in \operatorname{SmP}(K)$. As $X \rightarrow \operatorname{spec}(K)$ extends to a smooth projective morphism $f: X \rightarrow U$ over a dense open subscheme $U \hookrightarrow S$, such a $V_{\ell}$ actually arises as the stalk of a subquotient $\mathcal{V}_{\ell}$ of the $\overline{\mathbb{Q}}_{\ell}$-local system $R^{i} f_{*} \overline{\mathbb{Q}}_{\ell}(j)$. In particular, for every closed point $s \in|S|$ with residue field $k(s)$, the inverse characteristic polynomial $\operatorname{det}\left(1-T F_{S} \mid \mathcal{V}_{\ell, \bar{s}}\right)$ of the geometric Frobenius $F_{S}$ at $s$ divides $P_{i, j, s}(T):=\operatorname{det}\left(1-T F_{s} \mid H^{i}\left(X_{\bar{s}}, \overline{\mathbb{Q}}_{\ell}(j)\right)\right.$ which, according to the Weil conjectures, has coefficients in $\mathbb{Z}$ and is independent of $\ell$, and its roots are $k(s)$-Weil numbers of weight $i-2 j$. Since the number field $Q_{s}$, generated by the roots of $P_{i, j, s}$ is a degree $\leq \operatorname{deg}\left(P_{i, j, s}\right)$ ! extension of $\mathbb{Q}$, unramified outside $p$, and since $\operatorname{deg}\left(P_{i, j, s}\right)=\operatorname{dim} H^{i}\left(X_{\bar{s}}, \overline{\mathbb{Q}}_{\ell}(j)\right)$ is independent of $s$, by Hermite-Minkowski, there are only finitely many possibilities for $Q_{s}$ as $s$ varies in the (infinite!) set $|S|$. This a fortiori implies that the field $Q_{v_{e}}$ (usually called the field of coefficients or the field of traces of $\mathcal{V}_{\ell}$ ), generated by the coefficients of $\operatorname{det}\left(1-T F_{S} \mid \mathcal{V}_{\ell, \bar{s}}\right)$ when $s$ varies in $|S|$, is a number field. Morally, $Q_{V_{e}}$ is the field of definition of the motive $V \in \operatorname{Mot}_{k} \otimes_{\mathbb{Q}} \overline{\mathbb{Q}}$ from which $V_{\ell}$ arises. Now, if we fix another prime $\ell^{\prime} \neq p$, one can consider the $\ell^{\prime}$-adic realization $V_{\ell^{\prime}}=H_{\ell^{\prime}}(V)$. Again, the construction of $\operatorname{Mot}_{K}$ shows that (up to replacing $S$ by a dense open subscheme which only depends on $V$ and not on $\ell$ ) $V_{\ell^{\prime}}$ also arises as the stalk of a $\overline{\mathbb{Q}}_{\ell^{\prime}}$-local system $V_{\ell^{\prime}}$ on $S$ which is compatible with (or a companion / camarade of) $\mathcal{V}_{\ell}$ in the sense that

$$
\operatorname{det}\left(1-T F_{s} \mid \mathcal{V}_{\ell, \bar{s}}\right)=\operatorname{det}\left(1-T F_{s} \mid \mathcal{V}_{\ell^{\prime}, \bar{s}}\right), \quad s \in|S| .
$$


So far, what we explained is that a simple object $V_{\ell}$ in $\mathcal{T}_{H_{\ell}}^{\text {ess }} \otimes \overline{\mathbb{Q}}_{\ell}$ with finite determinant (to get rid of Tate twists) should arise (after possibly shrinking $S$ ) as the stalk of a simple $\overline{\mathbb{Q}}_{\ell}$-local system $\mathcal{V}_{\ell}$ with finite determinant on $S$ satisfying the following properties:

[Purity] the roots of $\operatorname{det}\left(1-T F_{s} \mid \mathcal{V}_{\ell, \bar{s}}\right)$ are $k(s)$-Weil numbers of weight $0, s \in|S|$;

[Finiteness] $Q_{\nu_{\ell}}$ is a number field;

[Companion] for every prime $\ell^{\prime} \neq p$ there is a $\overline{\mathbb{Q}}_{\ell^{\prime}}$-local system $V_{\ell^{\prime}}$ on $S$ which is compatible with $\mathcal{V}_{\ell}$.

The companion conjecture predicts that every simple $\overline{\mathbb{Q}}_{\ell^{-}}$ local system on $S$ with finite determinant behaves as if it were, indeed, arising from the $\ell$-adic realization of a motive! Namely,

Conjecture 1 (Companion; [Del80, (1.2.10)]). Let $\mathcal{V}_{\ell}$ be a simple $\overline{\mathbb{Q}}_{\ell}$-local system with finite determinant on $S$. Then $\mathcal{V}_{\ell}$ satisfies the above properties [Purity], [Finiteness], and [Companion].

So, if $\operatorname{Rep}_{\bar{Q}_{\ell}}\left(\pi_{1}(K)\right)^{s s, u r} \subset \operatorname{Rep}_{\bar{Q}_{\ell}}\left(\pi_{1}(K)\right)^{s S}$ denotes the full subcategory of $\pi_{1}(K)$-representations which are unramified over a dense open subscheme of $S$, what, in essence, the combination of the standard conjectures, the Tate conjecture, and the companion conjecture suggests very strongly is that the $\ell$-adic realization functor $\bar{H}_{\ell}$ : $\operatorname{Mot}_{K} \otimes_{\mathbb{Q}} \overline{\mathbb{Q}}_{\ell} \rightarrow \operatorname{Rep}_{\overline{\mathbb{Q}}_{\ell}}\left(\pi_{1}(K)\right)^{s s, u r}$ is an equivalence of categories. But while the standard and the Tate conjectures are still widely open, Conjecture 1 is now a theorem. The proof of Conjecture 1 runs over more than fourty years with decisive contributions by several authors such as Deligne, Drinfeld, and L. Lafforgue.

A few words about the proof. When $S$ is a curve, Conjecture 1 follows almost directly from the Langlands correspondence for $G L_{r}$ and the Ramanujan-Petersson conjecture. To put it in a nutshell, if $\mathrm{A}_{K}$ denotes the ring of adèles of $K$, the former predicts that there exists a canonial bijection $\pi \rightarrow \sigma_{\pi} / \pi_{\sigma} \leftarrow \sigma$ between isomorphism classes of automorphic cuspidal representations $\pi$ of $G L_{r}\left(\mathbb{A}_{K}\right)$ and of simple rank-r representations $\sigma$ in $\operatorname{Rep}_{\bar{Q}_{\ell}}\left(\pi_{1}(K)\right)^{s s, u r}$ characterized by the fact that $\pi$ and $\sigma_{\pi}$ have the same local L-factors, while the latter predicts that the Hecke eigenvalues of $\pi$ are $k$-Weil numbers of weight 0 . So in the context of the Langlands program, the part of motives is played by automorphic representations. This is not only an analogy since the general strategy to prove instances of the Langlands correspondence is to construct an algebraic stack $X$ over $K$ whose compactly supported $\overline{\mathbb{Q}}_{\ell}$ cohomology $H_{c}\left(X_{\bar{K}}, \overline{\mathbb{Q}}_{\ell}\right)$ is naturally equipped with an action of $G L_{r}\left(\mathbb{A}_{K}\right)$ commuting with the one of $\pi_{1}(K)$ and realizes the correspondence in the sense that there exists a family $\pi \otimes \sigma_{\pi}$ of simple $G L_{r}\left(\mathbb{A}_{K}\right) \times \pi_{1}(K)$-constituents of $H_{c}\left(X_{\bar{K}}, \overline{\mathbb{Q}}_{\ell}\right)$, indexed by the cuspidal automorphic representations $\pi$ of $G L_{r}\left(\mathbb{A}_{K}\right)$ with $\pi$ and $\sigma_{\pi}$ having the same local L-factors. One expects those $\pi \otimes \sigma_{\pi}$ to be cut out by algebraic correspondences on $H_{c}\left(X_{\bar{K}}, \overline{\mathbb{Q}}_{\ell}\right)$. The first breakthrough is due to Drinfeld, who proved the Langlands correspondence for $G L_{2}$ in the late 70 s and introduced the stack of chtoucas to play the part of $X$, and it is probably Drinfeld's work that led Deligne to formulate Conjecture 1. Drinfeld's proof was later generalized by L. Lafforgue to $r \geq 2$, who also proved the RamanujanPetersson conjecture [Laf02].

The proof of Conjecture 1 when $S$ is a curve is "almost motivic" in the sense that it exhibits a potential candidate-the stack of chtoucas-from which one should be able to construct explicitly the motive supporting $\sigma$. In constrast, for higher dimensional $S$, there is currently no such constructive strategy to prove Conjecture 1 and, just as for the Riemann hypothesis, the solution eventually came from a combination of geometric arguments, relying on the curve case and the theory of weights developed in [Del80]. The basic strategy is to show that a (semisimple) $\overline{\mathbb{Q}}_{\ell}$-local system $\mathcal{V}$ on $S$ can be reconstructed from the collection of its restrictions $\psi^{*} \mathcal{V}$ to smooth irreducible curves $\psi: C \rightarrow S$ together with the obvious glueing conditions $\left.\left.\psi^{*} \mathcal{V}\right|_{C{ }_{X} C^{\prime}} \simeq \psi^{\prime} * \mathcal{V}^{\prime}\right|_{C \times_{X} C^{\prime}}$. This is how Deligne completed the proof of [Purity] and [Finiteness] in Conjecture 1 [Del12] and how, building on this, Drinfeld completed the proof of [Companion] [Dri12]. Along the way, Deligne also proved that there are-up to twistsonly finitely many simple $\overline{\mathbb{Q}}_{\ell}$-local systems on $S$ and that their number is independent of $\ell$.

Beyond $\overline{\mathbb{Q}}_{\ell}$-local systems. The original formulation of Conjecture 1 also included a vaguely formulated $p$-adic part "on espère des petits camarades cristallins." And indeed, on top of $\ell$-adic cohomology, one has now at disposal other natural Weil cohomology theories for varieties over fields of characteristic $p>0$-in particular crystalline cohomology (with coefficients in $\overline{\mathbb{Q}}_{p}$ ) and ultraproduct cohomology (with coefficients in the ultraproducts $\overline{\mathbb{Q}}_{\mathfrak{u}}$, that is, the quotients of $\prod_{\ell \neq p} \overline{\mathbb{F}}_{\ell}$ by its non-principal maximal ideals $\mathfrak{u}$ ) with natural notions of local systems. As predicted by the motivic picture drawn above, Conjecture 1 extends for $p$-adic and ultraproduct local systems (except for the existence of $\overline{\mathbb{Q}}_{p}$-companions) by Abe, Abe-Esnault, and Kedlaya in the p-adic setting and Cadoret in the ultraproduct setting. This generalized form of Conjecture 1 makes it possible to transfer properties of local systems from one cohomological realm to the other. For instance, Cadoret-Tamagawa used [Companion] to reduce the Tannakian form of the Cebotarev density theorem for $\overline{\mathbb{Q}}_{p}$ - and $\overline{\mathbb{Q}}_{\mathfrak{u}}$-local systems to the (classical) Cebotarev density theorem for $\overline{\mathbb{Q}}_{\ell}$-local systems [CT20]. In a slightly different spirit, using the fact that rigid $\mathbb{C}$-local systems on a variety 
$X$ over $\mathbb{C}$ can be "specialized" to $\overline{\mathbb{Q}}_{\ell}$-local systems on reductions of $X$ over finite fields and a beautiful counting argument based on [Companion], Esnault-Groechenig proved Simpson's integrality conjecture for cohomologically rigid C-local systems [EG18].

\section{References}

[CT20] Anna Cadoret and Akio Tamagawa, A Tannakian Cebotarev density theorem and companions, 2020.

[Del74] Pierre Deligne, La conjecture de Weil. I (French), Inst. Hautes Études Sci. Publ. Math. 43 (1974), 273-307. MR340258

[Del80] Pierre Deligne, La conjecture de Weil. II (French), Inst. Hautes Études Sci. Publ. Math. 52 (1980), 137-252. MR601520

[Del12] Pierre Deligne, Finitude de l'extension de $\mathbb{Q}$ engendrée par des traces de Frobenius, en caractéristique finie (French, with French and Russian summaries), Mosc. Math. J. 12 (2012), no. 3, 497-514, 668, DOI 10.17323/1609-45142012-12-3-497-514 MR3024820

[Dri12] Vladimir Drinfeld, On a conjecture of Deligne (English, with English and Russian summaries), Mosc. Math. J. 12 (2012), no. 3, 515-542, 668, DOI 10.17323/1609-45142012-12-3-515-542. MR3024821

[EG18] Hélène Esnault and Michael Groechenig, Cohomologically rigid local systems and integrality, Selecta Math. (N.S.) 24 (2018), no. 5, 4279-4292, DOI 10.1007/s00029-0180409-z, MR3874695

[FM95] Jean-Marc Fontaine and Barry Mazur, Geometric Galois representations, Elliptic curves, modular forms, \& Fermat's last theorem (Hong Kong, 1993), Ser. Number Theory, I, Int. Press, Cambridge, MA, 1995, pp. 41-78. MR1363495

[Kle68] S. L. Kleiman, Algebraic cycles and the Weil conjectures, Dix exposés sur la cohomologie des schémas, Adv. Stud. Pure Math., vol. 3, North-Holland, Amsterdam, 1968, pp. 359-386. MR292838

[Laf02] Laurent Lafforgue, Chtoucas de Drinfeld et correspondance de Langlands (French, with English and French summaries), Invent. Math. 147 (2002), no. 1, 1-241, DOI 10.1007/s002220100174. MR1875184

[Wei49] André Weil, Numbers of solutions of equations in finite fields, Bull. Amer. Math. Soc. 55 (1949), 497-508, DOI 10.1090/S0002-9904-1949-09219-4. MR29393

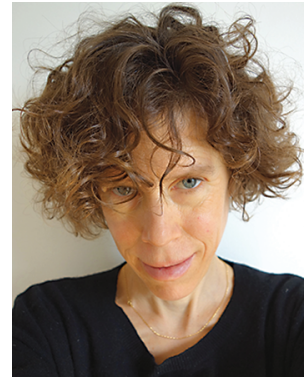

Anna Cadoret

\section{Credits}

Photo of Anna Cadoret is courtesy of Anna Cadoret.

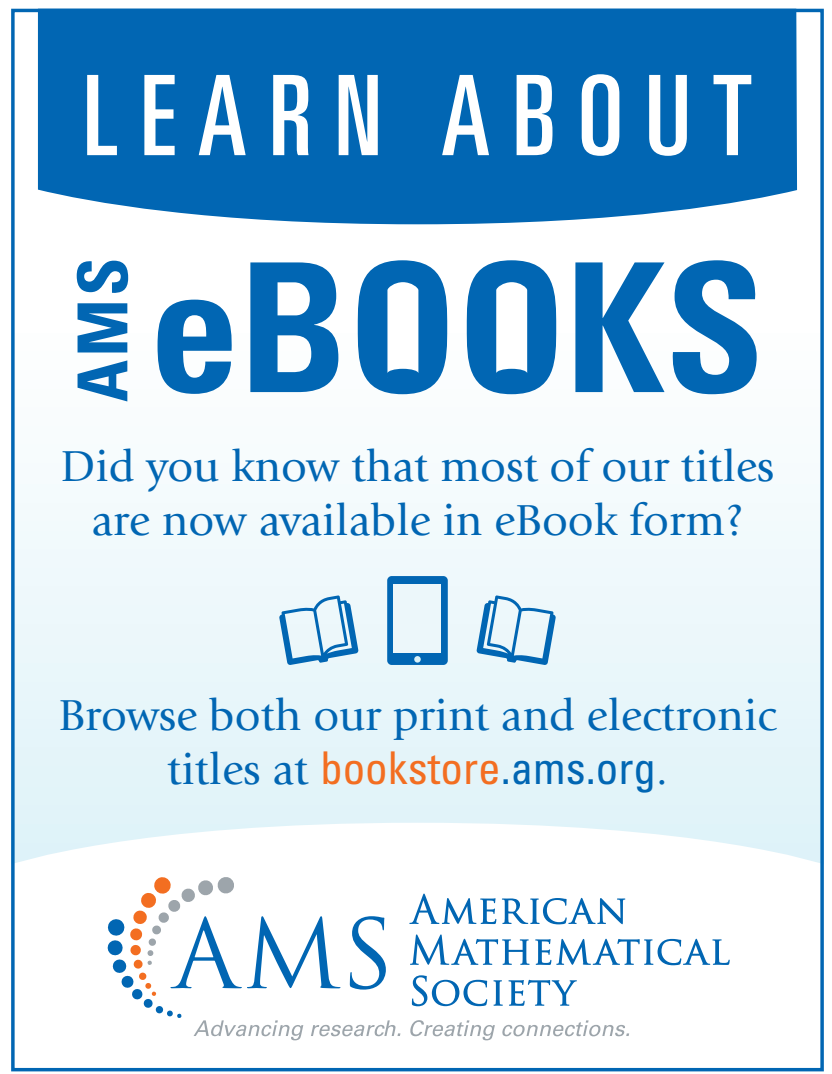

